\title{
Adding a reward increases the reinforcing value of fruit
}

\author{
Nathalie De Cock $^{1 *}$, Leentje Vervoort ${ }^{2}$, Patrick Kolsteren ${ }^{1}$, Lieven Huybregts ${ }^{3}$, Wendy Van Lippevelde ${ }^{4}$, \\ Jolien Vangeel $^{5}$, Melissa Notebaert ${ }^{5}$, Kathleen Beullens ${ }^{5,6}$, Lien Goossens ${ }^{2}$, Lea Maes ${ }^{3}$, \\ Benedicte Deforche ${ }^{4,7}$, Caroline Braet $^{2}$, Steven Eggermont ${ }^{5}$, John Van Camp ${ }^{1}$ and Carl Lachat ${ }^{1}$ \\ ${ }^{1}$ Department of Food Safety and Food Quality, Ghent University, Coupure Links 653, Ghent, Belgium \\ ${ }^{2}$ Department of Developmental, Personality and Social Psychology, Ghent University, Henri Dunantlaan 2, Ghent, Belgium \\ ${ }^{3}$ Poverty, Health and Nutrition Division, International Food Policy Research Institute, $2033 \mathrm{~K}$ Street, Washington, DC 20006, USA \\ ${ }^{4}$ Department of Public Health, Ghent University, De Pintelaan 185A, Ghent, Belgium \\ ${ }^{5}$ Leuven School for Mass Communication Research, KU Leuven, Parkstraat 45 - Box 3603, Leuven, Belgium \\ ${ }^{6}$ Research Foundation-Flanders (FWO), Egmontstraat 5, Brussels, Belgium \\ ${ }^{7}$ Physical Activity, Nutrition and Health Research Unit, Faculty of Physical Education and Physical Therapy, Vrije Universiteit \\ Brussel, Brussels, Belgium
}

(Submitted 30 August 2016 - Final revision received 12 December 2016 - Accepted 31 January 2017)

\section{Abstract}

Adolescents' snack choices could be altered by increasing the reinforcing value (RV) of healthy snacks compared with unhealthy snacks. This study assessed whether the RV of fruit increased by linking it to a reward and if this increased RV was comparable with the RV of unhealthy snacks alone. Moderation effects of sex, hunger, BMI $z$-scores and sensitivity to reward were also explored. The RV of snacks was assessed in a sample of 165 adolescents (15.1 (sD 1.5) years, 39.4\% boys and 17.4\% overweight) using a computerised food reinforcement task. Adolescents obtained points for snacks through mouse clicks (responses) following progressive ratio schedules of increasing response requirements. Participants were (computer) randomised to three experimental groups (1:1:1): fruit ( $n$ 53), fruit + reward ( $n$ 60) or unhealthy snacks ( $n$ 69). The RV was evaluated as total number of responses and breakpoint (schedule of terminating food reinforcement task). Multilevel regression analyses (total number of responses) and Cox's proportional hazard regression models (breakpoint) were used. The total number of responses made were not different between fruit + reward and fruit $(b-473 ; 95 \% \mathrm{CI}-1152,205, P=0 \cdot 17)$ or unhealthy snacks ( $b$ 410; $95 \% \mathrm{CI}-222,1043, P=0 \cdot 20$ ). The breakpoint was slightly higher for fruit than fruit + reward (HR 1.34; $95 \% \mathrm{CI} 1.00,1 \cdot 79, P=0.050$ ), whereas no difference between unhealthy snacks and fruit + reward (HR 0.86; $95 \%$ CI 0.62, 1.18, $P=0.34$ ) was observed. No indication of moderation was found. Offering rewards slightly increases the RV of fruit and may be a promising strategy to increase healthy food choices. Future studies should however, explore if other rewards, could reach larger effect sizes.

Key words: Reinforcing value: Fruits: Snacks: Rewards: Adolescents

The overconsumption of energy-dense snacks contributes to excess energy intake in adolescents ${ }^{(1,2)}$. Consumption of energydense snacks is primarily driven by hedonic processes such as food reinforcement rather than by homoeostatic motives ${ }^{(3,4)}$. The reinforcing value (RV) of a food, or the motivation to eat, is usually assessed as the amount of work an individual is willing to perform to gain access to that food ${ }^{(4)}$. A higher RV of energy-dense snacks is associated with increased energy intake and an increased risk of obesity in children, adults and adolescents ${ }^{(5-8)}$. Unhealthy energydense snacks, such as chocolate and chips, have a higher RV than healthy snacks, such as fruit and vegetables, driving individuals towards unhealthy snack choices ${ }^{(9,10)}$.
Behavioural choice theory suggests that the consumption of unhealthy snacks can be decreased by either decreasing the $\mathrm{RV}$ of unhealthy snacks or by increasing the RV of alternatives or substitutes ${ }^{(9-12)}$. To date, most research has focused on decreasing the RV of unhealthy snacks. Increasing the cost to obtain unhealthy snacks shifted choice towards healthy snacks in children and adults ${ }^{(9,10)}$. The effect of increasing the RV of healthy snacks has not been assessed. Following the principles of operant conditioning, one might assume that adding a reward to the choice for fruit or other healthy snacks could be one possible strategy to increase the RV of healthy snacks in adolescents $^{(13-15)}$. Offering rewards or praise has already

Abbreviations: BAS DRV, BAS drive; FRT, food reinforcement task; HR, hazard ratio; PR, progressive ratio; RV, reinforcing value; SR, sensitivity to reward; zBMI, BMI z-scores.

* Corresponding author: N. De Cock, email nathaliel.decock@ugent.be 
been shown to enhance children's willingness to taste and consumption of healthy food items such as fruit ${ }^{(14-17)}$. However, little is known about using reward-based strategies to promote healthy food consumption in adolescents. Such strategies are particularly relevant to evaluate in adolescents as they are highly susceptible to rewards and show higher activity in the reward-related brain regions compared with children and adults ${ }^{(18,19)}$. Therefore the first aim of the present study was to assess whether the RV of fruit could be increased by linking fruit with a reward (RV fruit + reward $v$. RV fruit alone). Second, we investigated whether the RV of fruit + reward was then comparable with the RV of unhealthy snacks (RV fruit + reward $v$. unhealthy snacks).

Previous research has shown that the RV of food is influenced by individual characteristics such as $\operatorname{sex}^{(3,20)}$, weight ${ }^{(4,5,8)}$ and hunger $^{(3)}$. The RV of unhealthy snacks was found to be higher in hungry or obese participants, whereas the RV of caffeinated beverages was found to be higher in males ${ }^{(3-5,8,20)}$. Differences in hunger, sex and weight might also be related to the difference in RV of unhealthy and healthy foods ${ }^{(3,10,21,22)}$. Hunger might only be associated with an increased RV of energy-dense snacks, whereas the RV of low-energy snacks such as fruit remain unchanged ${ }^{(3)}$. Obese or overweight individuals and boys found energy-dense and not low-energy dense snacks more reinforcing compared with their leaner peers or girls $^{(3,10,21,22)}$. A higher sensitivity to reward (SR), a psychobiological personality trait defined as one's ability to experience pleasure or reward on exposure to appetitive stimuli such as palatable foods ${ }^{(23)}$, might also be associated with a higher RV of palatable foods. Consistent with this idea, SR was found to be associated with preferences for unhealthy snack intakes in children and adolescents ${ }^{(24,25)}$. Individual differences in SR were already found to influence the use of rewards. Children with a high SR were more likely to taste healthy foods when rewarded $^{(15)}$. High SR adolescents might thus show a higher RV for fruit + reward compared with fruit alone. The third aim of the present study was to explore whether the difference in RV between fruit + reward and unhealthy snacks or fruit was influenced by sex, BMI, hunger or SR.

\section{Methods}

This study was conducted in the context of the REWARD project, which aims to improve snacking habits of adolescents using a novel framework. REWARD combines reward sensitivity theory with behaviour choice and learning theories, and focuses on the rewarding value of food and individual differences in SR to change behaviour. Guided by the results of the present study, a reward-based intervention to improve adolescents' snack choices delivered through a game will be developed.

\section{Participants and study design}

A convenience sample of 14-16-year-old adolescents from five secondary schools in the vicinity of Ghent, Belgium participated in this study in November 2015. The school principal of each of the five schools selected one to five classes to participate in the present study. All students from fourteen classes $( \pm 15$ students per class) from the five schools were invited to participate. No exclusion or inclusion criteria were used. Participants were randomly allocated using a computer-generated sequence to one of three experimental groups (1:1:1). Participants were blinded to the group allocation, while research assistants were blinded to the study hypotheses.

To detect a difference of $25 \%$ in RV (total number of responses made) between three parallel-allocated experimental groups and possible interactions with a power of $80 \%$ a sample size of 159 adolescents was needed (PASS software version 14; NCSS). Taking into account a possible non-participation due to absence, the anticipated sample size was increased to 210 students.

\section{Study procedures}

Participants completed the experiment together with their classmates in the school computer classroom on a weekday from 09.30 to 10.30 hours (around the morning school break), from 14.30 to 15.30 hours (around the afternoon school break) or from 15.30 to 16.30 hours (just before the end of the school day), as these are typical times during which adolescents consume snacks ${ }^{(26)}$. Participants were asked to eat and drink normally, but to abstain from eating or drinking (except water) for at least $2 \mathrm{~h}$ before the experimental session. At the beginning of the session participants were provided with a choice of two isoenergetic preloads (sandwich with ham or cheese, $\pm 753 \mathrm{~kJ}$ $( \pm 180 \mathrm{kcal}))$. The consumption of this standard preload diminishes the effects of hunger on food reinforcement and increases the ability of observing individual differences in food reinforcement $^{(27)}$. After eating this preload, adolescents started the experiment. Half of the participants started the experiment with the general questionnaire and the height/weight measurements; while the other half, the adjacent sitting participants, started with the computerised food reinforcement task (FRT) to measure the RV of food and the hunger questionnaire. Adolescents completed the FRT to gain points to trade for fruit (experimental group 1), unhealthy snacks (experimental group 2) or fruit + reward (experimental group 3) at the end of the task. Participants could choose the fruit or unhealthy snacks they wanted to earn points for. The five fruits options were: grapes, apple, pear, plum or tangerine and the five unhealthy snacks were: candy bar, chocolate, marshmallows, cookies or potato crisps. Adolescents in the fruit + reward group were informed that not only could they earn points to receive fruit portions at the end of the task, but also that the person with the highest number of points obtained could become the class winner. This message was displayed on a specific slide during the introduction of the FRT and was only visible to the fruit + reward group. The other two experimental groups were unaware of the competition and were only informed that their points gathered in the FRT would earn them fruit or unhealthy snack portions at the end of the task. The possibility to become the class winner through a competition was chosen as reward, as intangible rewards are thought to not disturb intrinsic motivation ${ }^{(28)}$ and competition and winning appeals to youngsters, especially in a game context $^{(29,30)}$. Before the experiment, participants were told that the study intended to examine participant's abilities to 
concentrate on a monotonous task and that this task would be different for everyone. After the experiment, adolescents were informed about the actual purpose and design of the study.

\section{Ethics}

Active written informed consent forms and study information folders for the parents were distributed a few days before the study commencement and collected during the test. Before the test, adolescent participants were also asked to compile a written informed consent form. This study was conducted according to the guidelines laid down in the Declaration of Helsinki and all procedures involving human subjects were approved by the ethics committee of the Ghent University Hospital.

\section{Measures}

Both the general and hunger questionnaires were online questionnaires and administered on a computer. The general questionnaire assessed the individual characteristics of the participants and the hunger questionnaire the hunger feeling of the participants before the FRT.

\section{Individual characteristics}

Both sex and date of birth were assessed with one-item questions. Age was then derived by subtracting the date of birth from the date the survey took place.

Consumption frequency of snacks was measured with a oneitem question 'How often do you normally consume a snack?' according to the following four categories: $1=$ once a week or less, $2=$ more than once a week, $3=$ every day and $4=$ more than once a day.

SR was measured using the BAS drive (BAS DRV) subscale of the Dutch child version of Carver and White's BIS/BAS scale ${ }^{(31)}$. This scale consists of four items, scored on a four-point scale ( $1=$ not at all true, $2=$ somewhat not true, $3=$ somewhat true, $4=$ all true) and summed to obtain the BAS DRV score, with a higher score indicating more SR (range 4-16). This BAS DRV subscale was chosen to measure SR as previous research in children, adolescents and adults had already shown that mainly BAS DRV was associated with food intake and eating styles $^{(32-34)}$ and that it is a valid instrument to measure SR in children and adolescents ${ }^{(35,36)}$. Internal consistency of the BAS DRV score in the present sample was good (Cronbach's $\alpha=0 \cdot 83$ ).

Height and weight were measured by two trained research assistants using a standardised protocol. Adolescents were measured wearing light clothing and without shoes. Body height was measured with a Leicester Portable Stadiometer (SECA) with an accuracy of $1 \mathrm{~mm}$. Weight was measured with a calibrated electronic scale SECA 861 with an accuracy of $100 \mathrm{~g}$. Age and sex-specific BMI $z$-scores (zBMI) were calculated using Flemish 2004 growth reference data ${ }^{(37)}$.

Hunger before the experiment was measured by a one-item question 'How hungry do you feel at the moment?', evaluated on a seven-point Likert scale with anchors $1=$ 'not hungry at all' and $7=$ 'extremely hungry ${ }^{(4,8)}$.

\section{Food reinforcement task}

The RV of the different snack foods was measured using a FRT with a progressive ratio (PR) schedule. At the beginning of the FRT, participants received a brief introduction on the screen informing them that they could earn points to trade for food by clicking the mouse button (response) and that increasingly more responses would be needed to obtain points. Subsequently adolescents in fruit + reward group additionally received the competition message on the screen. After this introduction and according to the allocated experimental group, the participants chose which specific unhealthy snack or fruit item they wanted to trade earned points for through the FRT. After indicating their preference, participants started the FRT. Points were earned according to a PR schedule that began at 2 (called PR2) and progressed through PR4, PR8, PR16, PR32, PR64, PR128, PR256 and PR512. In the first schedule (PR2), the participants gained 1 point for each second response, in the second level (PR4) participants gained 1 point after four responses and so on. When 20 points were obtained, the participant progressed to the next PR schedule. When participants were no longer motivated to work for food, they terminate the task by pressing the space bar. To avoid satiation and/or habituation, participants only received their food portions earned after they had decided to terminate the task. Participants were informed (during the introduction) that for each point earned, they either received $10 \mathrm{~g}$ of fruit or $5 \mathrm{~g}$ of unhealthy snacks (depending on their allocated experimental group) at the end of the task. Twice as many points were needed to obtain the same amount of unhealthy snacks compared with fruit, because a meaningful portion of fruit (e.g. a tangerine) usually weighs more than a meaningful portion of the unhealthy snacks (e.g. a handful of potato crisps). Similar to previous studies that assessed the RV of food using PR schedules ${ }^{(4,38)}$, the outcomes of the experiment were the total number of responses made across all PR schedules (total number of mouse button clicks) and the breakpoint or the PR schedule, where the adolescent decided to terminate the FRT (schedule of terminating the FRT).

\section{Statistical analyses}

First, the difference in the total number of responses made (dependent variable) between the experimental groups and the subsequent moderation analyses were assessed using a multilevel linear regression model with two levels (adolescents nested within classes) to account for the clustering. Our analysis strategy entailed the computation of six models. Model 1 was an intercept-only model without any level 1 or level 2 independent variables. Model 2 evaluated the effect of the experimental group, which was added as a categorical independent variable with three categories (fruit + reward = reference category, fruit and unhealthy snacks). Models 3-6 evaluated the possible moderation effects of sex, zBMI, hunger or SR in separate models by adding the moderator and the interaction moderator $\mathrm{X}$ experimental group as independent variables to model 2. Continuous parameters were mean centred, unstandardised coefficients and their standard errors were reported 
and associations with $P$-values $<0.05$ were considered statistically significant. As the total number of responses was positively skewed, square root transformations (best-fitting transformation) were applied to produce a normal distribution. The findings both for the raw and the square root transformed data were similar and hence the analyses of the raw data were presented to facilitate interpretation.

Second, the difference in the breakpoint (dependent variable) between the experimental groups and the subsequent moderation by SR, sex, zBMI or hunger were assessed using survival analysis. Cox proportional hazards models were used to model the schedule reached when terminating the FRT (breakpoint). Censoring was applied when adolescents reached the end of the FRT (PR512), however no participant actually reached this schedule. In model 1 the hazard ratios (HR) of fruit $v$. fruit + reward and unhealthy snacks $v$. fruit + reward were computed and the estimated survival curves for each experimental group were plotted. For instance, a HR of 1.2 for fruit $v$. fruit + reward indicates that at any given FR schedule, the risk of terminating the computer task is 1.2 times higher for fruit than fruit + reward. Models 2 until 5 assessed moderation effects of SR, sex, zBMI or hunger before the experiment. Separate models were developed by adding the moderator and the interaction term moderator X experimental group to model 1 as independent variables. Schedule of reinforcement reached was recoded to represent time until they stopped responding as follows: PR2 =1, PR4=2, PR8 =3, PR16=4, PR32 =5, PR64=6, PR $128=7$, PR256 $=8$ and PR512 $=9$. Standard errors and CI of the coefficients were adjusted for possible dependency of participants/observations within a class by using a clustered sandwich estimator. The Breslow's method was used to handle ties. The proportional hazards assumption that the hazard or risk remains constant over time was tested with the Grambsch and Thernay test of the Schoenfeld residuals ${ }^{(39)}$.

All analyses were conducted using Stata version 13 SE (StataCorp LP).

\section{Results}

\section{Participants}

Of the 210 selected adolescents, fourteen (6.7\%) were unable to participate due to school absence, thus 196 adolescents participated in the study. Of these 196 participating adolescents, 182 were randomised and completed the FRT (see Fig. 1); fourteen participants $(7 \cdot 1 \%$ of the 196$)$, who started with the general questionnaire, did not complete this questionnaire and therefore could not start the FRT; eighteen (9.9\% of the 182 randomised participants) participants, who started with the FRT, did not finish the general questionnaire. A total of 165 adolescents thus completed both the FRT and the general questionnaire and were included in the analysis (see Fig. 1). The mean age was 15.1 ( $\mathrm{sD} 1.5$ ) years, $39.4 \%$ were males. Of the adolescents $30 \cdot 3 \%$ ate a snack every day and $22.4 \%$ ate two or more snacks per day. Percentages or mean scores and standard deviations for age, snack frequency, sex, SR, hunger before the experiment, zBMI and total number of responses according to experimental group are presented in Table 1.

\section{Total number of responses made}

The intercept-only model (model 1 ) showed that overall, adolescents made an average of 2254 (SD 191) responses in the FRT (Table 2). Model 2, with experimental group as independent variable, indicated that there are no significant differences in total number of responses between the fruit + reward and the fruit-only $(P=0 \cdot 17)$ or the unhealthy snack $(P=0 \cdot 20)$ group. Adolescents in the fruit-only group made on average 473 (95\% CI -1152, 205) responses less than for fruit + reward and the unhealthy snacks group showed 410 (95\% CI -222, 1043) responses more compared with the fruit + reward group.

\section{Breakpoint}

The HR was marginally significantly higher for the fruit-only group compared with the fruit + reward group (Table 3). The risk of terminating the task at any schedule was 1.34 times higher when responding for fruit than for fruit + reward (HR 1.34; $95 \%$ CI 1.00, 1.79, $P=0.050$ ). The risk of terminating the task for participants of the unhealthy snacks group was similar to the risk in the fruit + reward group (HR 0.86; 95\% CI 0.62 , $1 \cdot 18, P=0 \cdot 34)$. The estimated survival function for each of the experimental groups is shown in Fig. 2.

\section{Moderation by sex, BMI Z-scores, hunger or sensitivity to reward}

For total responses made, no indication of moderation by sex, zBMI, hunger or SR was found $(P>0.05$ for all interaction terms, see Table 2 ). Model fit only significantly improved (compared with model 2) for the moderation models with zBMI (model 4) and hunger (model 5).

Similar to the breakpoint analyses, no moderation by sex, zBMI, hunger or SR was observed $(P>0.05$ for all interaction terms, see Table 3 ). The model fit only significantly improved (compared with model 1) for the moderation models with zBMI (model 3) and hunger (model 4).

\section{Discussion}

The present study investigated whether linking fruit with an intangible reward, could significantly increase the RV of fruit and if this observed increased RV was comparable with the RV of unhealthy snacks in an adolescent sample. The RV, in terms of breakpoint, of fruit + reward was found to be marginally higher by $34 \%$ than the RV of fruit and not significantly different from that of unhealthy snacks.

To date, no studies have evaluated the RV of fruit or unhealthy snacks in terms of breakpoint analyses. This is unfortunate as Bickel et al. ${ }^{(40,41)}$ showed that peak response measures, such as the total number of responses made, are less robust than breakpoint analyses to detect differences in RV between different reinforcers. Bickel found that the reinforcer (cigarettes $v$. money) that had the highest peak response varied across participants, while the reinforcer with the largest breakpoint was the same for all participants ${ }^{(41)}$. 


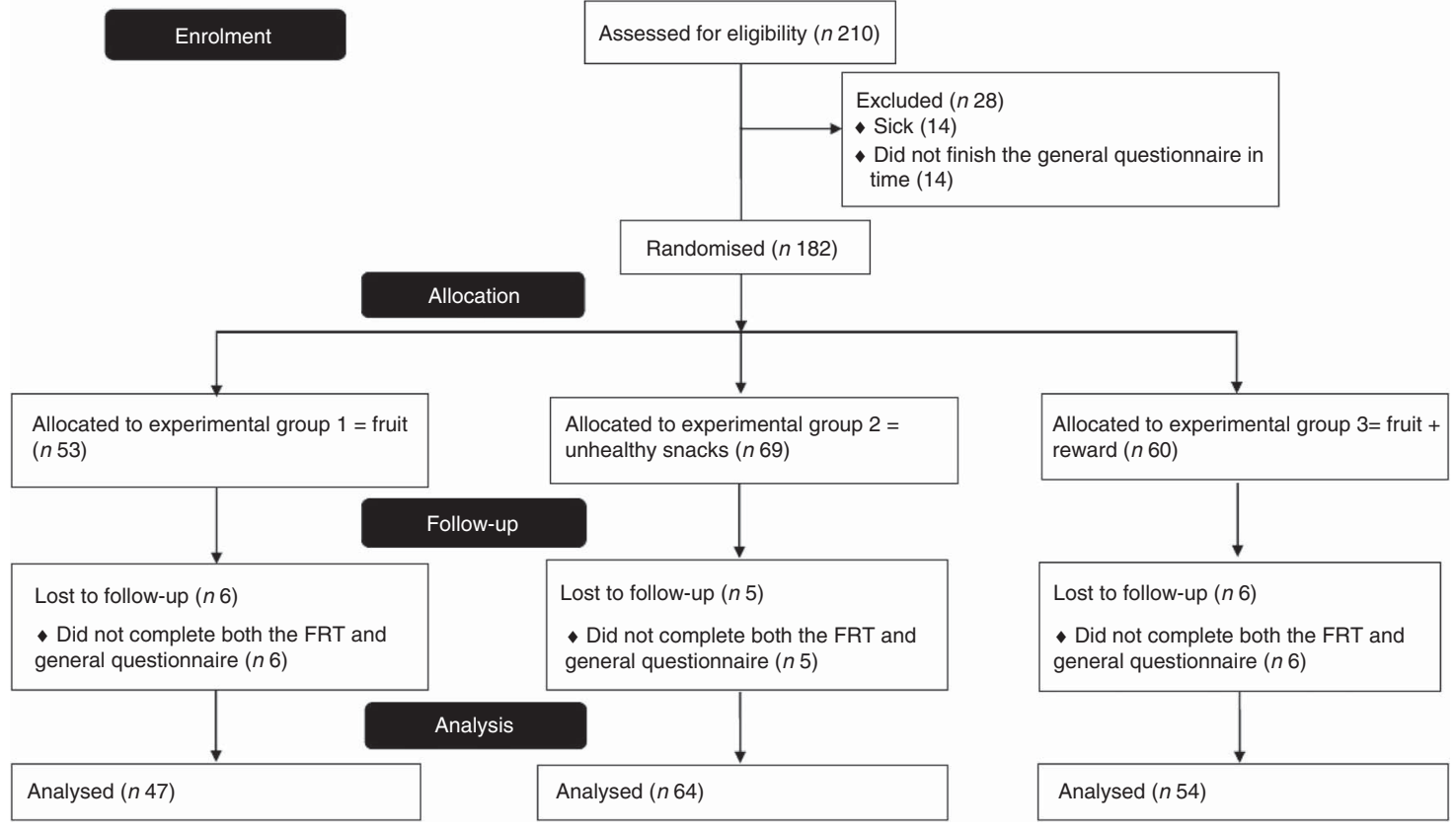

Fig. 1. Consort flow chart. FRT, food reinforcement task.

Table 1. Participant characteristics according to experimental group (Mean values and standard deviations)

\begin{tabular}{|c|c|c|c|c|c|c|}
\hline & \multicolumn{2}{|c|}{ Fruit ( $n$ 47) } & \multicolumn{2}{|c|}{ Fruit + reward ( $n$ 54) } & \multicolumn{2}{|c|}{ Unhealthy snacks ( $n$ 64) } \\
\hline & Mean & SD & Mean & SD & Mean & SD \\
\hline Boys (\%) & \multicolumn{2}{|c|}{40.4} & \multicolumn{2}{|c|}{38.9} & \multicolumn{2}{|c|}{39.1} \\
\hline Ate a snack each day (\%) & \multicolumn{2}{|c|}{$27 \cdot 7$} & \multicolumn{2}{|c|}{31.5} & \multicolumn{2}{|c|}{31.3} \\
\hline Ate two or more snacks per day (\%) & \multicolumn{2}{|c|}{$17 \cdot 0$} & \multicolumn{2}{|c|}{$24 \cdot 1$} & \multicolumn{2}{|c|}{$25 \cdot 0$} \\
\hline Age (years) & $15 \cdot 02$ & 0.84 & $15 \cdot 21$ & 0.87 & 15.02 & $2 \cdot 13$ \\
\hline Hunger feeling before the experiment $(1-7)$ & 3.12 & 1.68 & 3.53 & 1.43 & 3.28 & 1.52 \\
\hline zBMl & 0.41 & 0.96 & 0.13 & 0.92 & 0.38 & 0.91 \\
\hline SR (4-16) & 9.49 & 2.64 & 9.74 & 3.22 & 9.83 & 2.96 \\
\hline Total number of responses made & $1712 \cdot 68$ & $1412 \cdot 84$ & $2270 \cdot 93$ & 1853.91 & $2672 \cdot 88$ & 1822.66 \\
\hline
\end{tabular}

zBMI, BMI z-scores; SR, sensitivity to reward.

The breakpoint in the present study was marginally higher for fruit+reward group than for fruit-only group and not significantly different from unhealthy snacks. The HR of the fruit group was however $34 \%$ higher than for fruit + reward group and the unhealthy snacks group had HR that was 14\% lower than the fruit + reward group. Adolescents in the fruit + reward group therefore had $34 \%$ lower risk to stop responding at lower schedules of reinforcement. In other words, they were willing to do $34 \%$ more effort to obtain fruit than adolescents in the fruitonly group. To our knowledge, no other studies have evaluated the breakpoint by means of survival analysis. The present analysis, however, is favourable over traditional approaches that compare the mean breakpoint, as it allows assessing the chance (the risk) of terminating the FRT at each schedule. The latter is of particular interest as chances to terminate the FRT are usually smaller for low PR schedules and higher for high PR schedules ${ }^{(42)}$.

The RV, in terms of the total number of responses made, was not significantly different between the different experimental groups. The RV of fruit + reward was not significantly higher than fruit and not significantly different from unhealthy snacks. Adding a reward to fruit, the experiment diminished the difference in the total number of responses between fruit and unhealthy snacks by $38 \%$. Adolescents responded on average $56 \%$ more for unhealthy snacks than for fruit and only responded $18 \%$ more for unhealthy snacks than for fruit + reward. Although previous studies already compared the RV of fruit and unhealthy snacks in terms of total number of responses made, no other studies have investigated the possibility to increase the RV of fruit ${ }^{(9,10)}$. Previous experiments indicated that adults increased responses by $20^{(10)}$ or $15 \%^{(9)}$ for unhealthy snacks compared with fruit, given equal response requirements ${ }^{(9,10)}$. The smaller difference in RV observed compared with our study, maybe due to the fact that the latter studies evaluated the RV of fruit and unhealthy snacks relative to another, while we measured the absolute $\mathrm{RV}^{(4)}$. Epstein et al. ${ }^{(4)}$ states that the absolute and relative RV of foods are however, similar when the alternative presented during the experiment is not very reinforcing. The relative RV can be smaller than the absolute RV when the alternative itself is also reinforcing. 
Table 2. Effect of experimental group on the total number of responses made ( $b$ Values and $95 \%$ confidence intervals)

\begin{tabular}{|c|c|c|c|c|c|c|c|c|c|c|c|c|}
\hline & \multicolumn{2}{|r|}{ Model 1} & \multicolumn{2}{|r|}{ Model 2} & \multicolumn{2}{|r|}{ Model 3} & \multicolumn{2}{|r|}{ Model 4} & \multicolumn{2}{|r|}{ Model 5} & \multicolumn{2}{|r|}{ Model 6} \\
\hline & $b$ & $95 \% \mathrm{Cl}$ & $b$ & $95 \% \mathrm{Cl}$ & $b$ & $95 \% \mathrm{Cl}$ & $b$ & $95 \% \mathrm{Cl}$ & $b$ & $95 \% \mathrm{Cl}$ & $b$ & $95 \% \mathrm{Cl}$ \\
\hline Constant & $2253.68^{\star * *}$ & * $1879.95,2627.40$ & $2233 \cdot 29^{* \star *}$ & $1700 \cdot 76,2765 \cdot 81$ & $1993 \cdot 42^{\star \star \star}$ & $1235 \cdot 33,2751.51$ & $2135 \cdot 85^{\star \star *}$ & $1593.87,2677.83$ & $2199 \cdot 27^{\star \star *}$ & $1625 \cdot 87,2772 \cdot 66$ & $2234.69^{* * *}$ & $1707 \cdot 22,2762 \cdot 17$ \\
\hline Unhealthy snacks $v$. fruit + reward & & & 410.3719 & $-222 \cdot 41,1043 \cdot 16$ & 287.65 & $-685 \cdot 02,1260 \cdot 34$ & 510.95 & $-163.61,1185.52$ & 400.00 & $-221 \cdot 88,1021 \cdot 88$ & 401.48 & $-226 \cdot 17,1029 \cdot 13$ \\
\hline Fruit $v$. fruit + reward & & & $-473 \cdot 26$ & $-1151.94,205.41$ & $-326 \cdot 45$ & $-1367 \cdot 10,714 \cdot 20$ & -331.41 & $-1058 \cdot 31,395.48$ & -491.02 & $-1141 \cdot 76,159.72$ & -481.87 & $-1154 \cdot 94,191 \cdot 21$ \\
\hline Sex (girl $v$. boys) & & & & & 379.07 & $-535.48,1293.62$ & & & & & & \\
\hline Sex $\times$ snack & & & & & 220.92 & $-1037.25,1479.09$ & & & & & & \\
\hline Sex $\times$ fruit & & & & & -234.56 & $-1565.58,1096.46$ & & & & & & \\
\hline zBMI & & & & & & & -55.26 & $-576 \cdot 79,466 \cdot 26$ & & & & \\
\hline zBMI $\times$ snack & & & & & & & 58.19 & $-661.08,777.46$ & & & & \\
\hline zBMl $\times$ fruit & & & & & & & 136.83 & $-625 \cdot 47,899 \cdot 13$ & & & & \\
\hline Hunger & & & & & & & & & 72.97 & $-239 \cdot 55,385 \cdot 50$ & & \\
\hline Hunger $\times$ snack & & & & & & & & & 196.48 & $-208 \cdot 79,601 \cdot 75$ & & \\
\hline Hunger $\times$ fruit & & & & & & & & & 68.62 & $-346 \cdot 81,484.06$ & & \\
\hline SR & & & & & & & & & & & 70.90 & $-68.85,210 \cdot 66$ \\
\hline $\mathrm{SR} \times$ snack & & & & & & & & & & & 30.84 & $-166 \cdot 34,228.03$ \\
\hline $\mathrm{SR} \times$ fruit & & & & & & & & & & & -104.95 & $-335 \cdot 30,125 \cdot 40$ \\
\hline Log likelihood & & -1465.06 & & $-1461 \cdot 47$ & & $-1460 \cdot 21$ & & -1275.06 & & $-1244 \cdot 11$ & & -1459.93 \\
\hline $2 \Delta$ Log likelihood $(\Delta \mathrm{df}) \dagger$ & & $N / A$ & & N/A & 2.52 & 3 & $372 \cdot 82^{\star \star \star}$ & 3 & $434.72^{\star \star \star}$ & 3 & 3.08 & 3 \\
\hline
\end{tabular}

ZBMI, BMl Z-scores; SR, sensitivity to reward; N/A, not applicable.
${ }_{* \star *} P<0.001$.

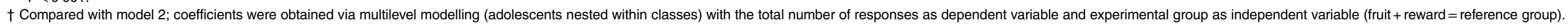


Table 3. Effect of experimental group on the breakpoint (Hazard ratios (HR) and $95 \%$ confidence intervals)

\begin{tabular}{|c|c|c|c|c|c|c|c|c|c|c|}
\hline & \multicolumn{2}{|c|}{ Model 1} & \multicolumn{2}{|c|}{ Model 2} & \multicolumn{2}{|c|}{ Model 3} & \multicolumn{2}{|c|}{ Model 4} & \multicolumn{2}{|c|}{ Model 5} \\
\hline & HR & $95 \% \mathrm{Cl}$ & $\mathrm{HR}$ & $95 \% \mathrm{Cl}$ & HR & $95 \% \mathrm{Cl}$ & HR & $95 \% \mathrm{Cl}$ & $\mathrm{HR}$ & $95 \% \mathrm{Cl}$ \\
\hline Unhealthy snacks $v$. fruit + reward & 0.86 & $0.62,1 \cdot 18$ & 0.95 & $0.58,1.55$ & 0.81 & $0.63,1.10$ & 0.83 & $0.63,1.10$ & 0.86 & $0.62,1.19$ \\
\hline Fruit $v$. fruit + reward & $1.34 \dagger$ & $1.00,1.79$ & 1.54 & $0.97,2.44$ & 1.22 & $0.89,1.67$ & $1 \cdot 36^{\star}$ & $1.03,1.79$ & 1.33 & $1.00,1.79$ \\
\hline Sex (girls $v$. boys) & & & 0.92 & $0.61,1.40$ & & & & & & \\
\hline Sex x snack & & & 0.85 & $0.40,1.78$ & & & & & & \\
\hline Sex $\times$ fruit & & & 0.81 & $0.44,1.46$ & & & & & & \\
\hline zBMI & & & & & 1.02 & $0.74,1.40$ & & & & \\
\hline zBMI × snack & & & & & 1.00 & $0.67,1.48$ & & & & \\
\hline zBMI × fruit & & & & & 0.88 & $0.63,1.24$ & & & & \\
\hline Hunger & & & & & & & 0.97 & $0.83,1.13$ & & \\
\hline Hunger $\times$ snack & & & & & & & 0.94 & $0.78,1.14$ & & \\
\hline Hunger $\times$ fruit & & & & & & & 0.99 & $0.83,1.17$ & & \\
\hline SR & & & & & & & & & $0.98^{*}$ & $0.96,1.00$ \\
\hline $\mathrm{SR} \times$ snack & & & & & & & & & 1.00 & $0.94,1.06$ \\
\hline$S R \times$ fruit & & & & & & & & & 1.01 & $0.93,1.11$ \\
\hline Log pseudo likelihood & \multirow{2}{*}{\multicolumn{2}{|c|}{$\begin{array}{c}-734.81 \\
\text { N/A }\end{array}$}} & \multicolumn{2}{|c|}{-733.83} & \multicolumn{2}{|c|}{$-621 \cdot 73$} & \multicolumn{2}{|c|}{$-611 \cdot 60$} & \multicolumn{2}{|c|}{-734.51} \\
\hline $2 \Delta$ Log pseudo likelihood $(\Delta d f) \ddagger$ & & & 1.96 & 3 & $226 \cdot 18^{\star \star \star}$ & 3 & $246 \cdot 42^{\star \star \star}$ & 3 & 0.60 & 3 \\
\hline
\end{tabular}

zBMI, BMI Z-scores; SR, sensitivity to reward; N/A, not applicable.

${ }^{*} P<0.05,{ }^{* \star *} P<0.001, \dagger P=0.050$.

‡ Compared with model 1 ; coefficients were obtained via Cox's proportional hazard modelling with schedule of terminating the task as dependent variable and experimental group as an independent variable (fruit + reward = reference group), robust SE were calculated with a clustered sandwich estimator.

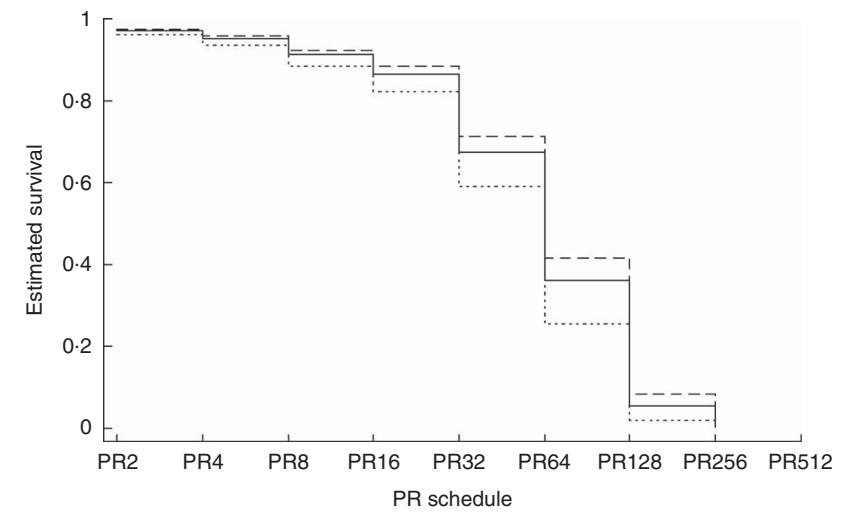

Fig. 2. Estimated survival function for each of the experimental groups. $\mathrm{PR}$, progressive ratio; estimated survival functions were obtained from the Cox's proportional hazard model with schedule of terminating the task as dependent variable and experimental group as independent variable (fruit + reward = reference group). - - Fruit + reward; -----, unhealthy snacks; -..-...-, fruit.

Vervoort et $a l .{ }^{(22)}$ also measured the absolute RV in adolescents, but found a larger difference in RV between fruit and unhealthy snacks compared with our study. The larger difference in the study by Vervoort et al. could be explained by the sequential design of the study as the RV of fruit and unhealthy snacks were measured in the same participants in sequential order. In the group that responded for unhealthy snacks first, adolescents responded $162 \%$ more for unhealthy snacks than for fruit; while in the group that worked for fruit first, adolescents responded $16 \%$ less for unhealthy snacks than for fruit ${ }^{(22)}$.

The RV of food is considered a good predictor of food choice, food consumption and obesity ${ }^{(4)}$. Therefore, our study suggests that offering intangible rewards may help to promote healthy food consumption. We thereby add to the findings from previous research conducted in children that using rewards may increase liking, wanting and consumption of healthy foods when used appropriately ${ }^{(15)}$. However, in this study we tested the RV of fruit + reward, fruit and unhealthy snacks as absolute, we did not take into account what would happen when an individual is presented with an actual choice between snack options $^{(43)}$. Both clinical (relative choice experiments) and field studies are still needed to further confirm our findings and to conclude that increasing the RV of fruit by rewarding strategies may change adolescents' snack choices. Within this study only a small effect size $(\mathrm{HR}>1 \cdot 3)^{(44)}$ was achieved for the breakpoint of fruit + reward $v$. fruit alone and both the breakpoint and total number of responses for unhealthy snacks were still larger than for fruit+reward. To maximise the chance that adolescents would actually favour healthy snacks over unhealthy snacks, the RV of fruit + reward should be further increased and other more potent type of rewards that could augment the RV of fruit should thus still be explored. Other studies have already showed that giving stickers increased fruit and vegetable intake on the short-term in children ${ }^{(45)}$ and that providing access to high-preference activities increased physical activity ${ }^{(46)}$. Strategies other than adding an additional reward to increase the RV of fruit should also be explored. The RV of fruit could also be altered starting from the principles of classical conditioning, by influencing adolescents' affective associations about fruit ${ }^{(13,47)}$. Previous research has shown that repeatedly pairing fruit stimuli (pictures of fruit) with positive stimuli (positive words or positive images), increased the chance of choosing fruit over unhealthy snacks when offered the choice ${ }^{(47)}$. Epstein et al., Vervoort et al. and Jacques-Tiura \& Greenwald also suggested that strategies to increase the RV of healthy foods should be combined with strategies to decrease the RV of unhealthy foods. This would increase the chances that people would alter their food choice and consumption habits ${ }^{(4,22,48)}$. Known methods to decrease the consumption of unhealthy snacks are to increase the costs (e.g. food taxing), to decrease the variety 
of unhealthy snack options and to decrease the portion size $\mathrm{e}^{(4,22,48,49)}$. To increase the consumption of healthy snacks methods other than rewards include subsidies, increasing variety of healthy snack options and making healthy snacks the default option in restaurants and cafeterias ${ }^{(4,22,48,49)}$.

In addition, it also known that individual characteristics influence the difference in RV of healthy foods and unhealthy foods ${ }^{(3,10,21,22)}$, the effect of rewarding strategies ${ }^{(15)}$ and in general the RV of food ${ }^{(3-5,8,20)}$. We therefore assessed whether the individual characteristics such as sex, BMI, state of hunger or SR moderated the difference in RV of fruit + reward and unhealthy snacks or fruit in adolescents. In the present study neither sex, zBMI, hunger nor SR significantly moderated the difference in RV between the fruit + reward and fruit or unhealthy snacks. To date, most research on the role of individual characteristics explaining differences in RV was carried out in children and adults, and focused solely on the RV of unhealthy snacks and not on the differences in RV between different alternatives $^{(3,4)}$. Only one other study researched the influence of individual characteristics (sex and SR) on the difference in RV of healthy and unhealthy snacks in adolescents ${ }^{(22)}$. Within this study also no moderation by SR could be documented, however a significant difference between boys and girls was found ${ }^{(22)}$. The difference in $\mathrm{RV}$ between fruit and unhealthy snacks was found to be larger for boys than girls ${ }^{(22)}$. As this is the first study that attempted to increase the RV of healthy snacks such as fruit, more research should be executed to further explore and confirm our findings that neither sex, BMI, the state of hunger or the SR influenced the difference in RV between fruit + reward and unhealthy snacks or fruit. Several additional individual characteristics such as restraint and habituation are also known to influence the RV of food in children and adults ${ }^{(3,50)}$, and are yet to be assessed in this regard.

This study is not without limitations. Adolescents completed the task together with their classmates in the same room. This set-up stimulated the desired competition feeling and made the possibility to be class winner realistic for the fruit + reward group. Nonetheless this set-up, also enabled interactions between the adolescents. The spillover effects were minimised as much as possible by the continuous presence of a researcher during the execution of the experiment. In addition, the order of completing the general questionnaire and the FRT was alternated for adjacent adolescents. Despite the fact that adolescents received a screen with snack choices according to their experimental group, it was possible that they observed differences in screens and thus realised that they were allocated to different groups. The researchers present in the room were also able to observe the different snack choice screens and were hence also not blinded to the allocation of the experimental groups. A discrepancy between the experimental setting and natural eating environments exists and generalisability to real life situations might be limited. However, experimentally measured RV has shown to have predictive validity for food intake and eating behaviour ${ }^{(4)}$. Several studies previously showed that the RV of foods measured in the laboratory is related to both laboratory energy intake and usual energy intake outside of the laboratory ${ }^{(7,51,52)}$. This experiment was primarily powered to detect an increase in $\mathrm{RV}$ from the fruit group. To ascertain equality of RV between the fruit + reward and unhealthy snacks however, an equivalence hypothesis is assumed. Post hoc power analysis in PASS 14 (NCSS) showed that equivalence could be detected in a sample of 110 adolescents ( $n 54$ for the fruit + reward group and $n 64$ for the unhealthy snacks group) with a power of $80 \%$ for a margin $(\Delta)$ of 900 responses. As this margin is more than double the actual observed difference between both groups, we are confident that adding reward to fruit increased RV to levels comparable with unhealthy snacks. The results of the present study are limited to 14-16-year-old adolescents, to a specific reward (class competition) and to a range of specific healthy and unhealthy snacks. More research is needed to extend the current findings to other age groups, rewards and types of snacks.

In conclusion, our results showed that linking an intangible reward to fruit increases the motivation to obtain fruit to an extent that it is comparable with the motivation to obtain unhealthy snacks. Offering rewards could thus be a promising strategy to increase healthy food choices, but it should still be tested in choice experiments and intervention studies whether or not combined with strategies to increase the cost of unhealthy foods. In addition future studies should also explore if other types of rewards, or other strategies to increase the RV of fruit, could reach larger effect sizes. Future research should also further explore the role of individual characteristics in light of the rewarding strategies proposed.

\section{Acknowledgements}

Data collection was assisted by several students: Annelies Malengier, Sander Vandamme, Elke Rammant, Floor De Groote, Lisa Schoenmaekers, Lisa Van Wilder en Eva Van de Velde.

This study was supported by the Agency for Innovation and Entrepreneurship of Flanders, Belgium (VLAIO). VLAIO had no role in the design, analysis or writing of this article. The first and corresponding author had access to all data at all times and had the final responsibility to submit the manuscript for publication.

The authors' responsibilities were as follows: N. D. C. conducted research, conducted the analyses and wrote the paper; L. V., C. L. and P. K. helped analysing the results and writing the paper; W. V. L., J. V. and M. N. conducted research and helped write the manuscript; L. H., L. G., K. B., S. E., B. D., L. M., J. V. C., C. B. designed research and helped revise the manuscript. All authors read and approved the final version of the manuscript.

The authors declare that there are no conflicts of interest.

\section{References}

1. Moreno LA \& Rodriguez G (2007) Dietary risk factors for development of childhood obesity. Curr Opin Clin Nutr Metab Care 10, 336-341.

2. Sebastian RS, Cleveland LE \& Goldman JD (2008) Effect of snacking frequency on adolescents' dietary intakes and meeting national recommendations. $J$ Adolesc Health 42, 503-511.

3. Temple JL (2014) Factors that influence the reinforcing value of foods and beverages. Physiol Behav 136, 97-103.

4. Epstein LH, Leddy JJ, Temple JL, et al. (2007) Food reinforcement and eating: a multilevel analysis. Psychol Bull 133, 884-906. 
5. Giesen JC, Havermans RC, Douven A, et al. (2010) Will work for snack food: the association of BMI and snack reinforcement. Obesity (Silver Spring) 18, 966-970.

6. Epstein LH, Yokum S, Feda DM, et al. (2014) Food reinforcement and parental obesity predict future weight gain in nonobese adolescents. Appetite 82, 138-142.

7. Epstein LH, Carr KA, Lin H, et al. (2011) Food reinforcement, energy intake, and macronutrient choice. Am J Clin Nutr $\mathbf{9 4}$ $12-18$.

8. Temple JL, Legierski CM, Giacomelli AM, et al. (2008) Overweight children find food more reinforcing and consume more energy than do nonoverweight children. Am J Clin Nutr 87, 1121-1127.

9. Giesen JC, Havermans RC \& Jansen A (2010) Substituting snacks with strawberries and Sudokus: does restraint matter? Health Psychol 29, 222-226.

10. Goldfield GS \& Epstein LH (2002) Can fruits and vegetables and activities substitute for snack foods? Health Psychol 21, 299-303.

11. Epstein LH \& Leddy JJ (2006) Food reinforcement. Appetite 46, 22-25.

12. Smith JA \& Epstein LH (1991) Behavioral economic-analysis of food choice in obese children. Appetite 17, 91-95.

13. Cooke LJ, Chambers LC, Anez EV, et al. (2011) Facilitating or undermining? The effect of reward on food acceptance. A narrative review. Appetite 57, 493-497.

14. Wardle J, Herrera ML, Cooke L, et al. (2003) Modifying children's food preferences: the effects of exposure and reward on acceptance of an unfamiliar vegetable. Eur J Clin Nutr 57, 341-348.

15. Vandeweghe L, Verbeken S, Moens E, et al. (2016) Strategies to improve the willingness to taste: the moderating role of children's reward sensitivity. Appetite 103, 344-352.

16. Lowe CF, Horne PJ, Tapper K, et al. (2004) Effects of a peer modelling and rewards-based intervention to increase fruit and vegetable consumption in children. Eur J Clin Nutr $\mathbf{5 8}$, 510-522.

17. Cooke LJ, Chambers LC, Anez EV, et al. (2011) Eating for pleasure or profit: the effect of incentives on children's enjoyment f vegetables. Psychol Sci 22, 190-196.

18. Hardin MG \& Ernst M (2009) Functional brain imaging of development-related risk and vulnerability for substance use in adolescents. I Addict Med 3, 47-54.

19. Galvan A (2013) The teenage brain: sensitivity to rewards. Curr Dir Psychol Sci 22, 88-93.

20. Temple JL, Bulkley AM, Briatico L, et al. (2009) Sex differences in reinforcing value of caffeinated beverages in adolescents. Behav Pharmacol 20, 731-741.

21. Clark EN, Dewey AM \& Temple JL (2010) Effects of daily snack food intake on food reinforcement depend on body mass index and energy density. Am J Clin Nutr 91, 300-308.

22. Vervoort L, Clauwaert A, Vandeweghe L, et al. (2016) Factors influencing the reinforcing value of fruit and unhealthy snacks. Eur J Nutr (epublication ahead of print version 25 August 2016).

23. Davis C, Patte K, Levitan R, et al. (2007) From motivation to behaviour: a model of reward sensitivity, overeating, and food preferences in the risk profile for obesity. Appetite 48, 12-19.

24. De Cock N, Van Lippevelde W, Vervoort L, et al. (2015) Sensitivity to reward is associated with snack and sugar sweetened beverage consumption in adolescents. Eur J Nutr 55, 1623-1641.

25. De Decker A, Sioen I, Verbeken S, et al. (2016) Associations of reward sensitivity with food consumption, activity pattern, and BMI in children. Appetite 100, 189-196.

26. Gevers DWM, Kremers SPJ, de Vries NK, et al. (2016) Intake of energy-dense snack foods and drinks among Dutch children aged 7-12 years: how many, how much, when, where and which? Public Health Nutr 19, 83-92.

27. Reiss S \& Havercamp S (1996) Sensitivity theory of motivation: implications for psychopathology. Behav Res Ther 34, 621-632.

28. Cameron J, Banko KM \& Pierce WD (2001) Pervasive negative effects of rewards on intrinsic motivation: the myth continues. Behav Anal 24, 1-44.

29. Baranowski T, Buday R, Thompson DI, et al. (2008) Playing for real - video games and stories for health-related behavior change. Am J Prev Med 34, 74-82.

30. Dickey MD (2005) Engaging by design: how engagement strategies in popular computer and video games can inform instructional design. EtrED 53, 67-83.

31. Muris P, Meesters C, Kanter E, et al. (2005) Behavioural inhibition and behavioural activation system scales for children: relationships with Eysenck's personality traits and psychopathological symptoms. Pers Indiv Differ 38, 831-841.

32. Verbeken S, Braet C, Lammertyn J, et al. (2012) How is reward sensitivity related to bodyweight in children? Appetite $\mathbf{5 8}$, 478-483.

33. De Cock N, Van Lippevelde W, Vervoort L, et al. (2016) Sensitivity to reward is associated with snack and sugarsweetened beverage consumption in adolescents. Eur J Nutr 55, 1623-1632.

34. Voigt DC, Dillard JP, Braddock KH, et al. (2009) Carver and White's (1994) BIS/BAS scales and their relationship to risky health behaviours. Pers Individ Dif 47, 89-93.

35. Muris P, Meesters C, De Kanter E, et al. (2005) Behavioural inhibition and behavioural activation system scales for children: relationships with Eysenck's personality traits and psychopathological symptoms. Pers Individ Dif $\mathbf{3 8}$, 103-113.

36. Vervoort L, Wolters LH, Hogendoorn SM, et al. (2010) Sensitivity of Gray's Behavioral Inhibition System in clinically anxious and non-anxious children and adolescents. Pers Individ Dif 48, 629-633.

37. Roelants M, Hauspie R \& Hoppenbrouwers K (2009) References for growth and pubertal development from birth to 21 years in Flanders, Belgium. Ann Hum Bio 36, 680-694

38. Roane HS (2008) On the applied use of progressive-ratio schedules of reinforcement. J Appl Behav Anal 41, 155-161.

39. Grambsch PM \& Therneau TM (1994) Proportional hazards tests and diagnostics based on weighted residuals. Biometrika 81, 515-526.

40. Bickel WK, Marsch LA \& Carroll ME (2000) Deconstructing relative reinforcing efficacy and situating the measures of pharmacological reinforcement with behavioral economics: a theoretical proposal. Psychopharmacology 153, 44-56.

41. Bickel WK \& Madden GJ (1999) A comparison of measures of relative reinforcing efficacy and behavioral economics: cigarettes and money in smokers. Behav Pharmacol 10, 627-637.

42. Hosmer DW, Lemeshow S \& May S (2008) Model development. In Applied Survival Analysis: Regression Modeling of Time-to-Event Data, 2nd ed., pp. 132-168. Hoboken, NJ: John Wiley and Sons, Inc.

43. Salvy SJ, Coelho JS, Kieffer E, et al. (2007) Effects of social contexts on overweight and normal-weight children's food intake. Physiol Behav 92, 840-846.

44. Bedard PL, Krzyzanowska MK, Pintilie M, et al. (2007) Statistical power of negative randomized controlled trials presented at American Society for Clinical Oncology annual meetings. J Clin Oncol 25, 3482-3487.

45. Lowe CF, Horne PJ, Hardman CA, et al. (2006) A peermodeling and rewards-based intervention is effective in 
increasing fruit and vegetable consumption in children. Prev Med 43, 351-351.

46. Goldfield GS, Lumb AB \& Colapinto CK (2011) Relative reinforcing value of energy-dense snack foods in overweight and obese adults. Can J Diet Pract Res 72, 170-174.

47. Walsh EM \& Kiviniemi MT (2014) Changing how I feel about the food: experimentally manipulated affective associations with fruits change fruit choice behaviors. J Behav Med 37, 322-331.

48. Jacques-Tiura AJ \& Greenwald MK (2016) Behavioral economic factors related to pediatric obesity. Pediatr Clin North Am 63, 425-446.
49. Huyghe E \& Van Kerckhove A (2013) Can fat taxes and package size restrictions stimulate healthy food choices? Int J Res Marketing 30, 421-423.

50. Epstein LH, Lin H, Carr KA, et al. (2012) Food reinforcement and obesity. Psychological moderators. Appetite 58, 157-162.

51. Epstein LH, Temple JL, Neaderhiser BJ, et al. (2007) Food reinforcement, the dopamine D2 receptor genotype, and energy intake in obese and nonobese humans. Behav Neurosci 121, 877-886.

52. Epstein LH, Wright SM, Paluch RA, et al. (2004) Relation between food reinforcement and dopamine genotypes and its effect on food intake in smokers. Am J Clin Nutr 80, 82-88. 